\title{
A novel predictive model based on inflammatory markers to assess the prognosis of patients with HBV-related acute-on-chronic liver failure: a retrospective cohort study
}

Li Qiang ${ }^{1}$, Jiao Qin², Changfeng Sun ${ }^{1}$, Yunjian Sheng ${ }^{1}$, Wen Chen ${ }^{1}$, Bangdong Qiu ${ }^{3}$, Xin Chen ${ }^{4}$, Yuanfang Chen ${ }^{1}$, Fei Liu' ${ }^{1}$ and Gang $\mathrm{Wu}^{1 *}$ (D)

\begin{abstract}
Background: Systemic inflammatory response is closely related to the development and prognosis of liver failure. This study aimed to establish a new model combing the inflammatory markers including neutrophil/lymphocyte ratio (NLR) and red blood cell distribution width (RDW) with several hematological testing indicators to assess the prognosis of patients with hepatitis B virus-related acute-on-chronic liver failure (HBV-ACLF).

Methods: A derivation cohort with 421 patients and a validation cohort with 156 patients were recruited from three hospitals. Retrospectively collecting their clinical data and laboratory testing indicators. Medcalc-15.10 software was employed for data analyses.

Results: Multivariate analysis indicated that RDW, NLR, INR, TBIL and Cr were risk factors for 90-day mortality in patients with HBV-ACLF. The risk assessment model is $C O X_{\text {RNTIC }}=0.053 \times R D W+0.027 \times N L R+0.003 \times \mathrm{TBIL}+0.317 \times$ INR $+0.003 \times \mathrm{Cr}$ (RNTIC) with a cut-off value of 3.08 (sensitivity: $77.89 \%$, specificity: $86.04 \%$ ). The area under the receiver operating characteristics curve (AUC) of the RNTIC was 0.873 [95\% CI(0.837-0.903)], better than the predictive value of MELD score [0.732, 95\% Cl(0.687-0.774)], MELD-Na [0.714, 95\% Cl(0.668-0.757)], CTP[0.703, 95\% $\mathrm{CI}(0.657-0.747)]$. In the validation cohort, RNTIC also performed a better prediction value than MELD score, MELD$\mathrm{Na}$ and CTP with the AUC of [0.845, 95\% Cl(0.778-0.898)], [0.768, 95\% Cl (0.694-0.832)], [0.759, 95\% Cl(0.684-0.824)] and $[0.718,95 \% \mathrm{Cl}(0.641-0.787)]$ respectively.
\end{abstract}

Conclusions: The inflammatory markers RDW and NLR could be used as independent predictors of 90-day mortality in patients with HBV-ACLF. Compared with MELD score, MELD-Na and CTP, RNTIC had a more powerful predictive value for prognosis of patients with HBV-ACLF.

Keywords: Liver failure, Hepatitis B virus, Prediction model, Inflammatory markers, Red blood cell distribution width, Neutrophil/lymphocyte ratio

\footnotetext{
*Correspondence: qiangli2019@126.com

'Department of Infectious disease, The Affiliated Hospital of Southwest

Medical University, Luzhou City 646000, Sichuan Province, China

Full list of author information is available at the end of the article
}

(C) The Author(s). 2020 Open Access This article is licensed under a Creative Commons Attribution 4.0 International License, which permits use, sharing, adaptation, distribution and reproduction in any medium or format, as long as you give appropriate credit to the original author(s) and the source, provide a link to the Creative Commons licence, and indicate if changes were made. The images or other third party material in this article are included in the article's Creative Commons licence, unless indicated otherwise in a credit line to the material. If material is not included in the article's Creative Commons licence and your intended use is not permitted by statutory regulation or exceeds the permitted use, you will need to obtain permission directly from the copyright holder. To view a copy of this licence, visit http://creativecommons.org/licenses/by/4.0/. The Creative Commons Public Domain Dedication waiver (http://creativecommons.org/publicdomain/zero/1.0/) applies to the data made available in this article, unless otherwise stated in a credit line to the data. 


\section{Background}

Acute-on-chronic liver failure (ACLF), a series of clinical syndrome resulted from culmination of chronic liver disease leading to single or multiple organ failures, has been shown to carry poor prognosis with a short-term mortality of $>50 \%$ [1]. At present, conservative medical treatment usually has been adopted, due to the artificial liver support system is poorly effective for end-stage liver failure, whereas stem cell therapy is still in development and faced with ethical issues [2]. Moreover, most patients with end-stage liver failure are suffering with multi-system organ failures resulting in many limitations in liver transplantation [3]. Therefore, reliable, userfriendly, inexpensive and reproducible predictors of survival are important to evaluate the risk of death early and choose treatment appropriately in those patients.

Currently, amounts of predictive scoring systems are available for assessing the prognosis in patients with ACLF, including chronic liver failure sequential organ failure assessment (CLIF-SOFA) score, Child-Turcotte Pugh (CTP) score, model for end-stage liver disease (MELD) score, MELD-sodium (MELD-Na) score [4]. The Model for End-Stage Liver Disease (MELD) score has the advantage of objective parameters which is often used for the prognosis of the patients with ACLF. In China, most cases of ACLF are caused by hepatitis B virus (HBV) infection, but those scores were established in European and American countries, where the alcohol is the most leading cause of the ACLF. Since these kinds of scoring systems might have certain limitations for HBV-related acute-on-chronic liver failure (HBV-ACLF), this study intended to establish a new model applicable to patients in China.

Nowadays, increasing evidences showed that systemic inflammatory response played a pivotal role in the development of liver failure and cirrhosis [5, 6]. A generalized activation of the inflammatory cytokines not only resulting to an accentuation of systemic circulatory dysfunction and organ hypo-perfusion, but also directly doing harm to organ function [7]. Inflammatory cytokines could affect the survival of erythrocytes, suppress maturation, lead larger and newer reticulocytes to enter circulation and increase the RDW [8]. The elevated granulocyte colony stimulating factorand granulocyte-macrophage colony stimulating factor, key regulatory cytokines that target committed progenitors promote differentiation and activation of monocytes and neutrophils [9]. Interleukin-6 (IL-6), an increased pro-inflammation cytokine in HBVACLF patients, also has ability to lead amounts of young platelets in the bone marrow to be released to the bloodstream [10] thus making the mean platelet volume (MPV) elevated [11]. The occurrence of ACLF generally represents a complicated state of host immune dysregulation. Excessive immune activation could lead to a decrease in lymphocyte numbers caused by activation induced cell death and impaired lymphopoiesis [12]. Based on the large amounts of investigations on systemic inflammation, routine hematology parameters, neutrophil/lymphocyte ratio (NLR), monocyte/lymphocyte ratio (MLR), platelet/ lymphocyte ratio (PLR), red cell distribution width (RDW), RDW/platelet ratio (RPR), gamma-glutamyl transpeptidase/platelet ratio (GPR), mean platelet volume (MPV), RDW/lymphocyte ratio (RLR) and prognostic nutritional index (PNI) and MPV/platelet ratio (MPR), are being considered as the inflammatory markers which could predict outcomes of various diseases [13-15]. Thus, this study aimed to identify inflammatory markers and hematological indicators associated with a short-term negative prognosis and establish a new multi-factor combined prognostic model for patients with HBV-ACLF.

\section{Methods}

\section{Patient selection}

ACLF was defined as the acute deterioration of liver function manifested as jaundice [total bilirubin (TBIL) $\geq 5 \mathrm{mg} / \mathrm{dL}$ or $\geq 85 \mu \mathrm{mol} / \mathrm{L}$ and coagulopathy with international normalized ratio of prothrombin time (INR) $\geq 1.5$ or prothrombin activity (PTA) $\leq 40 \%$, complicated with ascites and/or hepatic encephalopathy noted within 4 weeks in a patient diagnosed with HBV related chronic liver disease/cirrhosis [1]. All patients also meet the Chinese guidelines for diagnosis and treatment of liver failure [16]. The cirrhosis was diagnosed histologically proven or clearly considered on the basis of biological, clinical, and radiological features. Patients with cardiac diseases, endocrinological disorders, hematological disease and other types of cancer were excluded. Co-infection with human immunodeficiency virus, hepatitis A, C, D, and $E$ viruses or other hepatitis viruses, autoimmune diseases, alcoholic liver disease, acute liver failure, druginduced liver injury, coexistent hepatocellular carcinoma, and any other serious medical illness or patients who had received any immunotherapy, liver transplantation or artificial liver support were also excluded. The enrolled patients all hospitalized in the ward of infectious department, there were none patients on dialysis under mechanical ventilation.

421 patients admitted to the Affiliated Hospital of Southwest Medical University were consecutively recruited as a derivation cohort to establish the new prognostic model between January 1, 2014 to February 28, 2019. 56 patients in the First People's Hospital of Neijiang and 100 patients in the Second People's Hospital of Yibin from January 1, 2017 to February 28, 2019 were enrolled as a validation cohort. Retrospectively collecting their clinical data and baseline laboratory testing indicators and tracking patients' survivals by telephone and clinical follow-up until May 01, 2019 to 
ensure that the last case (enrolled before February 01, 2019) was also followed up the 90-day survival.

All patients admitted were given a standard medical treatment including nutritional support, antiviral therapy, intravenous infusion albumin and plasma, treatment of complications.

\section{Clinical data collection}

Clinical data included age, gender, telephone number, temperature, oxygen saturation, blood pressure, hepatic encephalopathy (HE), liver cirrhosis and type of infection were retrospectively abstracted from the medical record. The HE was diagnosed according to the West-Haven criteria [17]. Ascites and HE were the situation when patients were at admission. Bacterial infections were collected during the entire hospital stay. The diagnosis of bacterial infection was based on infection-positive cultures of blood, ascites, urine or sputum, and/or clinical symptoms suggestive of infections.

\section{Laboratory analysis}

Demographic and clinical characteristics of the included patients were recorded. Blood samples were collected from an antecubital vein after overnight fasting on the first day of admission, and detected the complete blood counts and biochemical tests by Mind 6800 automated blood analyzer and Mindray BS200 biochemical analyzer, respectively. Coagulation indicators were assessed using a CS-5100 automated coagulation analyzer. The HBVDNA levels in serum were quantified by ABI 7500FAST (fluorescence quantitative PCR). The above results were from the test departments of three hospitals with a high reliability. The MELD score was calculated using the Kamath formula: $\mathrm{R}=9.6 \times \ln (\mathrm{Cr} \mathrm{mg} / \mathrm{dl})+3.8 \times$ $\ln ($ TBIL $\mathrm{mg} / \mathrm{dl})+11.2 \times \ln (\mathrm{INR})+6.4$ [18]. PNI $=$ albu$\min (\mathrm{g} / \mathrm{L})+5 \times$ lymphocyte count $\left(10^{9} / \mathrm{L}\right)$. MELD-Na $=$ MELD+ $1.59 \times[135-\mathrm{Na}(\mathrm{mmol} / \mathrm{l})]$ in accordance with Biggins et al. [19]. CTP including HE, prothrombin time (PT), ascites, TBIL, and serum albumin was assessed according to the standard criteria [20].

\section{Statistical analysis}

Normally distributed variables were expressed as means \pm standard deviation (SD), and non-normally distributed variables were expressed as a median and interquartile range (IQR). Count and percentages were used to describe categorical variables. Two independent groups were compared using the $t$ test for continuous normally-distributed variables and the Mann-Whitney $U$ test for non-normally distributed variables. For categorical variables, comparisons between groups used the Chi-squared test or the Fisher test as appropriate. The Kaplan-Meier method was used to calculate the 90-day survival probability curves. The BOX-Tidwell method was used to test the wireless relationship between the independent and dependent variable. The tolerance and variance expansion factor were used to test the multicollinearity between the independent variables. Cox regression models were used for univariate and multivariate analysis of outcome predictors. Cut-off values were determined via the receiver operating characteristic (ROC) analysis. All calculations were performed by MedCalc software (version15.10). $P$ values $<0.05$ based on a two-tailed test were considered with statistically significance.

\section{Results}

Basic characteristics of patients with HBV-ACLF in derivation cohort

A total of $642 \mathrm{HBV}$-ACLF patients were collected from 3 centers. According to the inclusion and exclusion criteria, 65 patients were excluded (Fig. 1). Finally, 577 patients with HBV-ACLF were enrolled in the study. Among them, 421 patients from the affiliated hospital of southwest medical university were derivation cohort, and basic characteristics of patients were listed in Table 1.Besides, in the derivation cohort, 307 (72.92\%) patients complicated with bacterial infection, in whom $245(58.19 \%)$ had a single infection site, $41(9.73 \%)$ had 2 infection sites, and 21 (4.99\%) had $\geq 3$ infection sites on admission. The most frequent infection was pneumonia $(n=188,44.66 \%)$, followed by SBP $(n=167,39.67 \%)$ and intestinal infection $(n=36,8.55 \%)$.

\section{Comparison of inflammatory markers and routine hematological parameters between survivors and non- survivors}

In order to identify indicators with statistical differences, inflammatory markers and routine hematological parameters in the survivors and non-survivors were analyzed. For the inflammatory markers, compared with the survivors, the level of NLR, MLR, PLR, RLR and RDW increased $(P \leq 0.001)$ (Table 1$)$; For the routine hematological parameters, compared with the survivors, the serum $\mathrm{Na}$, PTA and lymphocytes were lower, while WBC, neutrophils, monocytes, TBIL, Cr, cyst-c, PT, INR, MELD scores, MELD-Na and CTP were higher $(P<0.05)$. Moreover, the incidence of hepatic encephalopathy was elevated in the non-survivors $(P<0.05)$ (Table 1$)$.

\section{Univariate and multivariate cox regression analysis of survival and death in HBV-ACLF patients}

Univariate regression analysis was performed on statistic significant indicators in Table 1, and multivariate cox regression analysis was performed on the indicators with significant difference in univariate analysis $(P<0.05)$, including TBIL, Cr, Cyst-c, INR, PTA, WBC, neutrophils, RDW, NLR, RLR, PLR, MLR. The multivariate cox regression results indicated that RDW, NLR, TBIL, INR, 


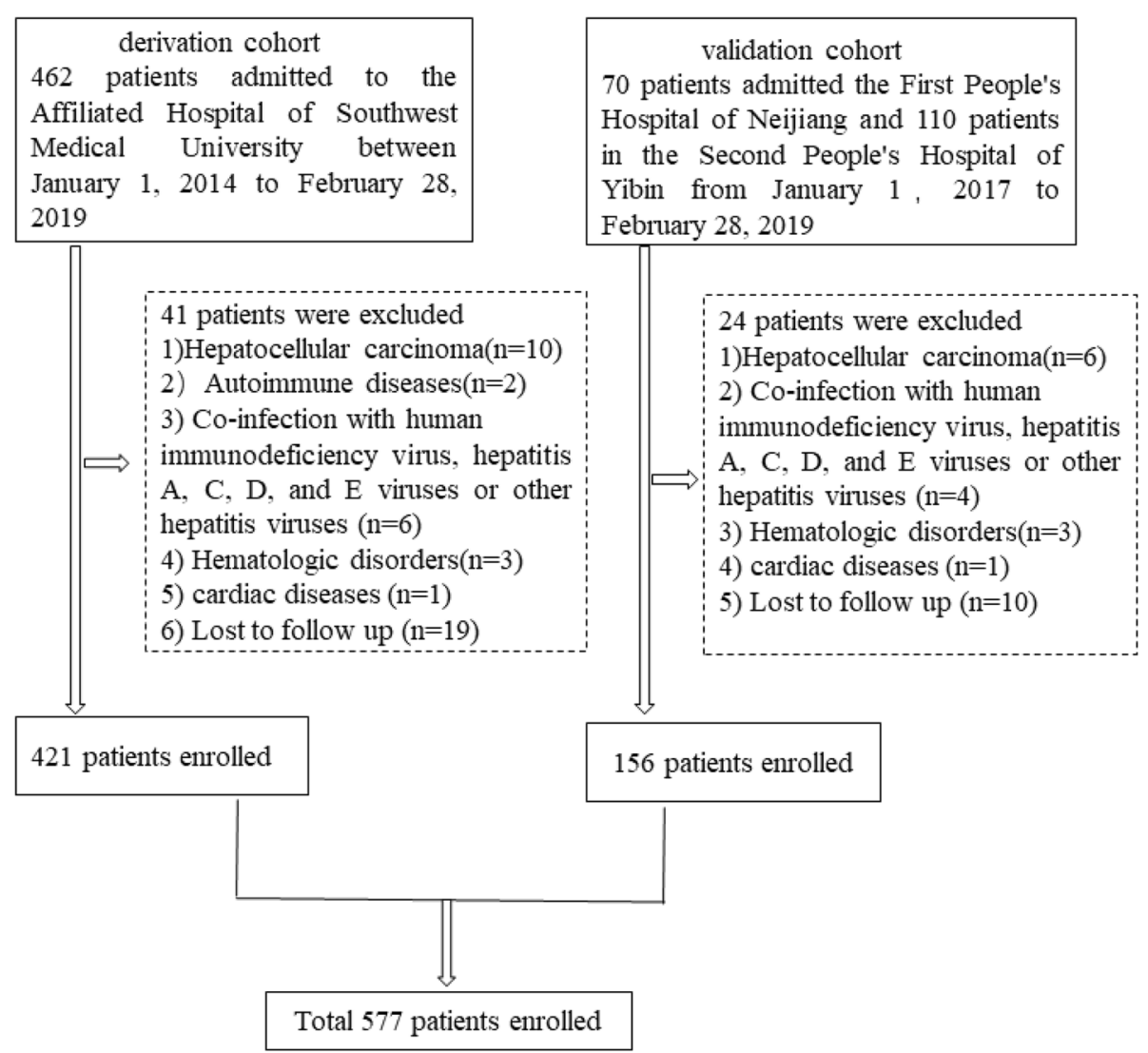

Fig. 1 A flow diagram of study participants

$\mathrm{Cr}$ were risk factors for 90-day death in HBV-ACLF patients $(P<0.05)$. In addition, RDW and NLR were significantly positively correlated with MELD scores $(P<$ 0.05), suggesting that high RDW, NLR might be closely associated with the prognosis of the patients with HBVACLF (Fig. 2a and b). We further identified the patients with HBV-ACLF based on the cut-off values of NLR and RDW to graph the Kaplan-Meier survival curves. The results showed that the patients with NLR $>4.09$ and RDW $>16.10$ had a more worse prognosis (Fig. 2c and d).

\section{Establishing a new prognostic model combining inflammatory markers with hematological parameters in patients with HBV-ACLF by cox regression}

The two inflammatory markers RDW, NLR and other three hematological parameters TBIL, INR, Cr had been found to be related to the prognosis of patients with HBV-ACLF in forward analysis. Based on the regression coefficient (Beta coefficient) as the weight of the risk factor (Table 2), the following model was established:

$\mathrm{COX}_{\mathrm{RNTIC}}=0.053 \times \mathrm{RDW}+0.027 \times \mathrm{NLR}+0.003 \times$

TBIL $+0.317 \times \mathrm{INR}+0.003 \times \mathrm{Cr}$ with a cut-off value of 3.08 (sensitivity: $77.89 \%$, specificity: $86.04 \%$ ). The model was able to predict 190 patients alive and 155 dead, accurately classifying $81.95 \%$ of the patients in this study (Table 3 ).

Comparison of predictive value of MELD score, MELD-Na, CTP and RNTIC for prognosis of patients with HBV-ACLF

Receiver operating characteristic (ROC) curves for parameters including MELD scores, MELD-Na, CTP and RNTIC were shown in Fig. 3a. RNTIC had a higher area under the ROC curve (AUC) for identifying poor prognosis than the other three $(p<0.001$, Table 3$)$.

\section{External validation of RNTIC}

In order to test the model, 180 patients were enrolled from the other two hospitals. According to the inclusion and exclusion criteria, 156 patients were admitted to the validation cohort (Fig. 1) with a 90-day mortality rate at $35.89 \%$. Comparisons of demographics and baseline clinical characteristics of the patients in the derivation and validation cohort were summarized in Table 4. The AUC of the RNTIC was higher than MELD, MELD-Na and CTP $(P<0.05$, Fig. 3b, Table 3), which proved this model also had an efficient ability on the prediction of the 90-day death in patients with HBV-ACLF in the validation cohort. 
Table 1 Comparisons of characteristics between survivors and non-survivors in patients with HBV-ACLF

\begin{tabular}{|c|c|c|c|c|}
\hline Variables & $\begin{array}{l}\text { Total patients } \\
(n=421)\end{array}$ & $\begin{array}{l}\text { Survivors } \\
(n=222)\end{array}$ & $\begin{array}{l}\text { Non-survivors } \\
(n=199)\end{array}$ & $P$ value \\
\hline Age (years) & $47.93 \pm 11.40$ & $48.10 \pm 11.20$ & $47.75 \pm 11.64$ & 0.735 \\
\hline Gender (M/F) & $365 / 56$ & $195 / 27$ & $170 / 29$ & 0.468 \\
\hline Cirrhosis (\%) & 299(71.02\%) & 159(71.62) & 140(70.35) & 0.744 \\
\hline Bacterial infections & $307(72.92 \%)$ & $145(65.32)$ & 162(81.41) & $<0.001$ \\
\hline HBV-DNA $\left(10^{7} \mathrm{IU} / \mathrm{mL}\right)$ & $1.53 \pm 4.85$ & $1.89 \pm 5.16$ & $1.17 \pm 4.53$ & 0.139 \\
\hline HbeAg(+) n (\%) & $116(27.55)$ & 67(30.18) & $49(24.62)$ & 0.203 \\
\hline $\mathrm{ALT}(\mathrm{U} / \mathrm{L})$ & $228(63.20,847.60)$ & $258.75(52.65,1000.33)$ & $198.20(74.80,755.10)$ & 0.532 \\
\hline $\mathrm{AST}(\mathrm{U} / \mathrm{L})$ & $223.40(103.95,641.90)$ & $254.10(94.63,254.10)$ & $206.10(110.10,511.20)$ & 0.477 \\
\hline TBIL $(\mu \mathrm{mol} / \mathrm{L})$ & $323.39 \pm 165.69$ & $236.35 \pm 118.55$ & $420.49 \pm 156.77$ & $<0.001$ \\
\hline $\mathrm{ALB}(\mathrm{g} / \mathrm{L})$ & $29.09 \pm 5.57$ & $29.33 \pm 5.81$ & $28.82 \pm 5.30$ & 0.348 \\
\hline$\gamma-G G T(U / L)$ & $85.40(48.5143 .00)$ & $79.35(48.45,157,13)$ & $86.30(48.50,126.80)$ & 0.719 \\
\hline $\mathrm{Cr}(\mu \mathrm{mol} / \mathrm{L})$ & $69.30(57.25,87.90)$ & $68.45(57.90,79.78)$ & $70.1(55.6106 .8)$ & 0.002 \\
\hline Cyst-c(mg/L) & $1.21(0.92,1.756)$ & $1.11(0.89,1.55)$ & $1.38(0.93,1.38)$ & 0.001 \\
\hline $\mathrm{K}^{+}(\mathrm{mmol} / \mathrm{L})$ & $3.97 \pm 0.65$ & $3.99 \pm 0.60$ & $3.95 \pm 0.70$ & 0.510 \\
\hline $\mathrm{Na}^{+}(\mathrm{mmol} / \mathrm{L})$ & $135.46 \pm 5.71$ & $135.79 \pm 5.70$ & $134.86 \pm 5.66$ & 0.042 \\
\hline PT(s) & $24.90(21.35,29.30)$ & $23.70(21.38,27.88)$ & $26.40(21.3,31.7)$ & 0.271 \\
\hline INR & $2.25(1.86,2.76)$ & $2.12(1.86,2.62)$ & $2.40(1.85,3.16)$ & $<0.001$ \\
\hline PTA (\%) & $34.11 \pm 10.60$ & $35.79 \pm 9.49$ & $32.23 \pm 11.45$ & 0.001 \\
\hline WBC $\left(10^{9} / \mathrm{L}\right)$ & $6.43(4.72,9.13)$ & $5.83(424,7.86)$ & $7.56(5.45,10.71)$ & $<0.001$ \\
\hline Neutrophils $\left(10^{9} / \mathrm{L}\right)$ & $4.65(3.11,7.21)$ & $3.92(2.88,5.80)$ & $5.98(3.82,8.52)$ & $<0.001$ \\
\hline Lymphocytes $\left(10^{9} / \mathrm{L}\right)$ & $0.96(0.66,1.33)$ & $1.03(0.71,1.41)$ & $0.89(0.61,1.28)$ & 0.030 \\
\hline Monocytes $\left(10^{9} / \mathrm{L}\right)$ & $0.61(0.40,0.90)$ & $0.54(0.37,0.77)$ & $0.70(0.45,1.04)$ & $<0.001$ \\
\hline RDW (\%) & $15.90(14.35,18.45)$ & $15.00(14.0,17.03)$ & $16.82(15.00,20.70)$ & $<0.001$ \\
\hline $\operatorname{PLT}\left(10^{9} / \mathrm{L}\right)$ & $86.00(58.0,126.0)$ & $82.00(53.0,125.0)$ & $89.00(64.0,89.0)$ & 0.155 \\
\hline$M P V(F L)$ & $11.75 \pm 1.53$ & $11.82 \pm 1.48$ & $11.67 \pm 1.58$ & 0.312 \\
\hline NLR & 4.84(3.19,8.09) & $4.00(2.57,6.10)$ & $6.31(4.19,10.25)$ & $<0.001$ \\
\hline GPR & $0.97(0.59,1.69)$ & $0.98(0.62,1.69)$ & $0.95(0.51,1.70)$ & 0.253 \\
\hline MLR & $0.64(0.42,0.94)$ & $0.53(0.38,0.74)$ & $0.77(0.52,1.10)$ & $<0.001$ \\
\hline RPR & $0.19(0.12,0.30)$ & $0.18(0.12,0.32)$ & $0.19(0.14,0.30)$ & 0.490 \\
\hline MPR & $0.14(0.09,0.20)$ & $0.15(0.10,0.22)$ & $0.13(0.09,0.19)$ & 0.103 \\
\hline $\mathrm{PNI}$ & $34.10(30.20,38.33)$ & $34.98(30.33,39.14)$ & $33.65(30.10,37.30)$ & 0.060 \\
\hline PLR & $91.03(62.66,127.75)$ & $85.20(57.45,118.76)$ & $102.15(70.37,144.12)$ & 0.001 \\
\hline RLR & $16.70(11.66,25.96)$ & $15.68(10.66,22.65)$ & $20.57(13.25,30.00)$ & $<0.001$ \\
\hline $\mathrm{PCT}(\mu \mathrm{g} / \mathrm{L})$ & $0.88(0.48,3.31)$ & $0.79(0.44,2.88)$ & $0.99(0.54,3.52)$ & 0.064 \\
\hline MELD SCORE & $25.05 \pm 6.75$ & $22.47 \pm 4.67$ & $27.94 \pm 7.52$ & $<0.001$ \\
\hline MELD-Na & $24.43 \pm 12.48$ & $20.36 \pm 11.09$ & $28.98 \pm 12.41$ & $<0.001$ \\
\hline CTP, n (\%) & & & & $<0.001$ \\
\hline $5-6$ & $1(0.24)$ & $1(0.45)$ & $0(0)$ & \\
\hline $7-9$ & 69(16.39) & $56(25.21)$ & $13(6.53)$ & \\
\hline$\geq 10$ & $351(83.37)$ & $165(74.32)$ & 186(93.46) & \\
\hline $\mathrm{HE}, \mathrm{n}(\%)$ & & & & 0.005 \\
\hline Stage 0 & $332(78.86)$ & 184(82.90) & 148(74.37) & \\
\hline Stage 1 & 14(3.33) & $9(4.10)$ & $5(2.51)$ & \\
\hline
\end{tabular}


Table 1 Comparisons of characteristics between survivors and non-survivors in patients with HBV-ACLF (Continued)

\begin{tabular}{llll}
\hline Variables & $\begin{array}{l}\text { Total patients } \\
(n=421)\end{array}$ & $\begin{array}{l}\text { Survivors } \\
(n=222)\end{array}$ & $\begin{array}{l}\text { Non-survivors } \\
(n=199)\end{array}$ \\
\hline Stage 2 & $23(5.46)$ & $12(5.4)$ & $11(5.53)$ \\
Stage 3 & $28(6.65)$ & $13(5.9)$ & $15(7.54)$ \\
Stage 4 & $24(5.70)$ & $4(1.8)$ & $20(10.05)$ \\
Antiviral therapy, $n(\%)$ & & \\
ETV & & $175(78.83)$ & $164(82.41)$ \\
TDF & $339(80.52)$ & $32(14.41)$ & $24(12.06)$ \\
LAM & $56(13.30)$ & $5(2.25)$ & $5(2.51)$ \\
ADV & $10(2.38)$ & $7(3.15)$ & $5(2.51)$ \\
ADV + LAM & $12(3.09)$ & $3(1.35)$ & $1(0.50)$
\end{tabular}

ALT alanine aminotransferase, AST aspartate aminotransferase, TBIL total bilirubin, ALB albumin, $\gamma$-GGT gamma-glutamyl transpeptidase, $C r$ creatinine, Cyst- $C$ cystatin c, Serum $k^{+}$serum potassium, Serum $\mathrm{Na}^{+}$serum sodium, $P T$ prothrombin time, INR international normalized ratio, PTA prothrombin activity, WBC white blood cell count, RBC red blood cells, HGB hemoglobin, PLT platelet, RDW red blood cell distribution width, NLR neutrophil/lymphocyte ratio, MLR monocyte/ lymphocyte ratio, $P L R$ platelet/lymphocyte ratio, RPR RDW/platelet ratio, GPR gamma-glutamyl transpeptidase/platelet ratio, MPV mean platelet volume, RLR RDW/ lymphocyte ratio, PNI prognostic nutritional index, MPR MPV/platelet ratio, PCT procalcitonin, MELD SCORE model for end-stage liver disease score, CTP childTurcotte Pugh score, MELD-Na MELD-sodium score, HE hepatic encephalopathy, ETV entecavir, TDF tenofovirdisoproxil, LAM lamivudine, ADV adefovir dipivoxil

\section{Discussion}

ACLF with a high mortality is a systemic inflammatory response driven by cytokines secretion, oxidative stress, immune dysfunction and increased risk of infection, which also compromises organ function integrity [21, 22]. In this study, a triple-center retrospective research was launched to create a new prognostic model taking inflammatory markers into consideration for patients with HBV-ACLF. Compared the routine hematological inflammatory parameters listed in Table 1, We found only NLR and RDW were independent prognostic factors associated with 90-day mortality in patients with HBV-ACLF, and then combined RDW and NLR with other three statistically significant indicators (TBIL, INR, Cr) to establish a new prognostic model, which performed a better predictive value both in derivation

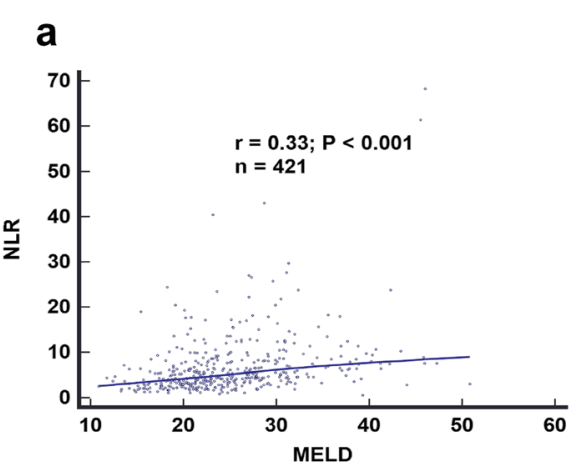

C

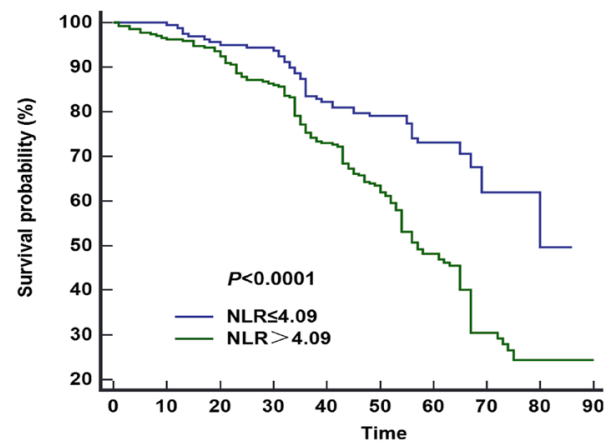

b

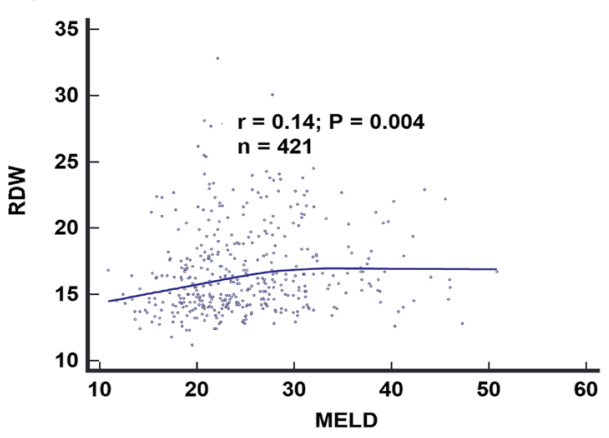

d

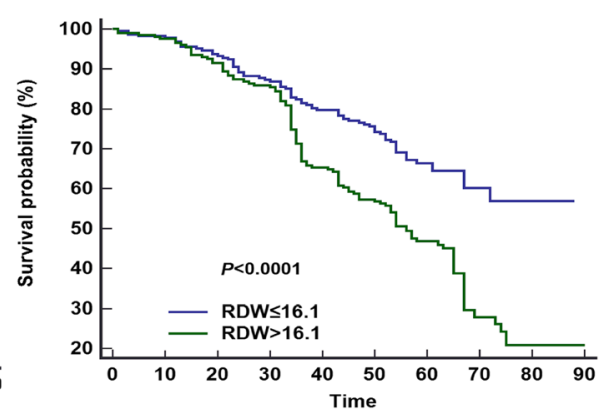

Fig. $2 \mathrm{NLR}(\mathbf{a})$ and RDW(b) levels correlated with MELD score in patients with HBV-ACLF, respectively; (c) The survival curves of groups of NLR>4.09 and NLR $\leq 4.09$ by Kaplan-Meier survival analysis; (d) The survival curves of groups of RDW>16.1 and RDW $\leq 16.1$ by Kaplan-Meier survival analysis 
Table 2 Cox regression analysis for variables associated with 90-day mortality in patients with HBV-ACLF

\begin{tabular}{|c|c|c|c|c|c|}
\hline \multirow[t]{2}{*}{ Variables } & \multicolumn{2}{|c|}{ Univariate analysis } & \multicolumn{3}{|c|}{ Multivariate analysis } \\
\hline & $\begin{array}{l}\mathrm{HR} \\
95 \%(\mathrm{Cl})\end{array}$ & $P$ value & Beta coefficient & $\begin{array}{l}\mathrm{HR} \\
95 \%(\mathrm{Cl})\end{array}$ & $P$ value \\
\hline TBIL $(\mu \mathrm{mol} / \mathrm{L})$ & $\begin{array}{l}1.005 \\
(1.004-1.005)\end{array}$ & $<0.001$ & 0.003 & $\begin{array}{c}1.002 \\
(1.001-1.003)\end{array}$ & $<0.001$ \\
\hline $\mathrm{Cr}(\mu \mathrm{mol} / \mathrm{L})$ & $\begin{array}{l}1.008 \\
(1.005-1.010)\end{array}$ & $<0.001$ & 0.003 & $\begin{array}{c}1.003 \\
(1.001-1.005)\end{array}$ & 0.001 \\
\hline Cyst-c (mg/L) & $\begin{array}{l}1.055 \\
(1.014-1.097)\end{array}$ & 0.008 & & & \\
\hline Serum $\mathrm{Na}^{+}(\mathrm{mmol} / \mathrm{L})$ & $\begin{array}{l}0.981 \\
(0.959-1.004)\end{array}$ & 0.112 & & & \\
\hline INR & $\begin{array}{l}1.488 \\
(1.342-1.651)\end{array}$ & $<0.001$ & 0.317 & $\begin{array}{c}1.318 \\
(1.163-1.494)\end{array}$ & $<0.001$ \\
\hline PTA (\%) & $\begin{array}{l}0.973 \\
(0.959-0.988)\end{array}$ & $<0.001$ & & & \\
\hline WBC $\left(10^{9} / \mathrm{L}\right)$ & $\begin{array}{l}1.068 \\
(1.043-1.094)\end{array}$ & $<0.001$ & & & \\
\hline Neutrophils $\left(10^{9} / \mathrm{L}\right)$ & $\begin{array}{l}1.082 \\
(1.055-1.110)\end{array}$ & $<0.001$ & & & \\
\hline Monocytes $\left(10^{9} / \mathrm{L}\right)$ & $\begin{array}{l}1.080 \\
(0.997-1.170)\end{array}$ & 0.060 & & & \\
\hline Lymphocytes $\left(10^{9} / \mathrm{L}\right)$ & $\begin{array}{l}0.856 \\
(0.640-1.144)\end{array}$ & 0.293 & & & \\
\hline RDW (\%) & $\begin{array}{l}1.112 \\
(1.075-1.15)\end{array}$ & $<0.001$ & 0.053 & $\begin{array}{c}1.047 \\
(1.009-1.086)\end{array}$ & 0.015 \\
\hline NLR & $\begin{array}{l}1.053 \\
(1.038-1.068)\end{array}$ & $<0.001$ & 0.027 & $\begin{array}{c}1.027 \\
(1.009-1.046)\end{array}$ & 0.003 \\
\hline MLR & $\begin{array}{l}1.057 \\
(1.007-1.109)\end{array}$ & 0.025 & & & \\
\hline PLR & $\begin{array}{l}1.003 \\
(1.001-1.005)\end{array}$ & 0.001 & & & \\
\hline RLR & $\begin{array}{l}1.016 \\
(1.007-1.026)\end{array}$ & 0.001 & & & \\
\hline \multirow[t]{2}{*}{ HE } & 1.221 & & & & \\
\hline & $(1.101-1.353)$ & $<0.001$ & & & \\
\hline \multirow[t]{2}{*}{ Bacterial infection } & 0.55 & & & & \\
\hline & $(0.383-0.785)$ & 0.001 & & & \\
\hline
\end{tabular}

TBIL total bilirubin, ALB albumin, $\mathrm{Cr}$ creatinine, Cyst-c Cystatin c, Serum $\mathrm{Na}^{+}$serum sodium, $P T$ prothrombin time, INR international normalized ratio, PTA prothrombin activity, WBC white blood cell count, $R D W$ red blood cell distribution width, NLR neutrophil/lymphocyte ratio, MLR monocyte/lymphocyte ratio, $P L R$ platelet/lymphocyte ratio, RLR RDW/lymphocyte ratio, HE Hepatic encephalopathy, HBV-ACLF hepatitis B virus related acute-on-chronic liver failure

and validation cohort. In addition, the study also analyzed the 28-day prediction ability of the inflammatory marker-based model. Compared with the prediction of 90-day viability, all models showed a poor ability to predict 28-day patient survival. Additional files showed this in more detail (see Additional files 1 and 2).

It was reported that increased neutrophil counts reflected oxidative stress and that lower lymphocyte counts reflected a deterioration of nutritional status [23]. Thus, the Neutrophil and lymphocyte counts could reflect inflammation status and general nutrition status of patients. The NLR has been researched in many diseases including liver disease. Increased NLR is predictive of mortality in advanced illnesses apart from infections including malignancy, acute coronary syndrome, intracerebral hemorrhage, chronic kidney disease and rheumatic diseases [24, 25], and elevated NLR has a tight relationship with the prognosis of hepatitis, liver cirrhosis and liver cancer $[14,26,27]$. In our study, the NLR value significantly elevated in the HBV-ACLF death group, and was an independent risk factor for 90-day death in HBV-ACLF patients, which was consistent with the study by Cai J, et al. [15], but the specific mechanism of HBV-ACLF patients' poor prognosis and NLR elevation is unclear. It was reported that in patients with end-stage liver disease, the body's immune system and 
Table 3 Comparison of predictive value of RNTIC, MELD, MELD-Na and CTP in derivation cohort and validation cohort

\begin{tabular}{|c|c|c|c|c|c|c|c|c|c|c|}
\hline Variables & $\begin{array}{l}\text { AUC } \\
(95 \%)\end{array}$ & Z Statistic & $P$ Value & Cut-off Value & $\begin{array}{l}\text { Sensitivity } \\
\text { (\%) }\end{array}$ & $\begin{array}{l}\text { Specificity } \\
(\%)\end{array}$ & $\begin{array}{l}\text { PPV } \\
(\%)\end{array}$ & $\begin{array}{l}\text { NPV } \\
(\%)\end{array}$ & $\begin{array}{l}\text { Overall } \\
\text { Accuracy } \\
(\%)\end{array}$ & $\begin{array}{l}\text { Youden } \\
\text { Index }\end{array}$ \\
\hline \multicolumn{11}{|c|}{ Derivation cohort } \\
\hline RNTIC & $\begin{array}{l}0.873 \\
(0.837-0.903)\end{array}$ & & & 3.08 & 77.89 & 86.04 & 82.89 & 81.2 & 81.95 & 0.64 \\
\hline MELD & $\begin{array}{l}0.732 \\
(0.687-0.774)\end{array}$ & 8.227 & $<0.001$ & 24.14 & 70.35 & 70.72 & 68.29 & 72.69 & 70.54 & 0.41 \\
\hline MELD-Na & $\begin{array}{l}0.714 \\
(0.668-0.757)\end{array}$ & 6.868 & $<0.001$ & 18.00 & 84.4 & 50.50 & 60.21 & 78.17 & 66.27 & 0.35 \\
\hline CTP & $\begin{array}{l}0.703 \\
(0.657-0.747)\end{array}$ & 6.424 & $<0.001$ & 10.00 & 82.91 & 47.30 & 58.51 & 75.54 & 64.13 & 0.30 \\
\hline \multicolumn{11}{|c|}{ Validation cohort } \\
\hline RNTIC & $\begin{array}{l}0.845 \\
(0.778-0.898)\end{array}$ & & & 2.59 & 85.07 & 71.00 & 76.25 & 80.26 & 78.21 & 0.62 \\
\hline MELD & $\begin{array}{l}0.768 \\
(0.694-0.832)\end{array}$ & 2.867 & 0.004 & 22.24 & 69.64 & 83.00 & 68.42 & 82.83 & 77.56 & 0.53 \\
\hline MELD-Na & $\begin{array}{l}0.759 \\
(0.684-0.824)\end{array}$ & 2.952 & 0.003 & 19.12 & 73.21 & 71.00 & 70.83 & 70.24 & 70.51 & 0.44 \\
\hline СТP & $\begin{array}{l}0.718 \\
(0.641-0.787)\end{array}$ & 2.903 & 0.003 & 10.00 & 75.00 & 63.00 & 65.82 & 68.83 & 67.31 & 0.38 \\
\hline
\end{tabular}

Z Statistic: compared with AUC of RNTIC, P value: compared with AUC of RNTIC, RNTIC $=0.053 \times \mathrm{RDW}+0.027 \times \mathrm{NLR}+0.003 \times \mathrm{TBIL}+0.317 \times \mathrm{INR}+0.003 \times \mathrm{Cr}, \mathrm{MELD}$ model for end-stage liver disease, CTP child-Turcotte Pugh score, MELD-Na MELD-sodium score, NLR neutrophil/lymphocyte ratio, RDW red blood cell distribution width, TBIL total bilirubin, $C r$ creatinine, INR international normalized ratio, HBV-ACLF hepatitis B virus related acute-on-chronic liver failure, NPV negative predictive value, $P P V$ positive predict value

inflammatory response were over-activated with a large number of inflammatory factors being released into the bloodstream (e.g., IL-6, IL-8, TNF- $\alpha$, etc.) [28], which caused damage to hepatocytes. Moreover, the robust inflammatory reaction could cause amounts of lymphocyte apoptosis, and make neutrophils originally presented in the hepatic sinusoids released into the blood, thereby increasing the level of NLR [29].Thus, the hypothesis that elevated NLR reflects the severity of the potentially acute systemic inflammation following primary injury is widely accepted.
In addition, another inflammatory marker RDW also was proved to be an independent risk factor for 90-day death in HBV-ACLF patients. However, the reason why RDW elevation is closely associated with the outcome of the patients with HBV-ACLF is still unclear. It may be due to the following five reasons: (I) Significant changes in RDW are associated with some abnormalities, such as inflammation, oxidative stress, red blood cell fragmentation, poor nutritional status, and erythropoietin dysfunction [30]. Pro-inflammatory factors could damage the
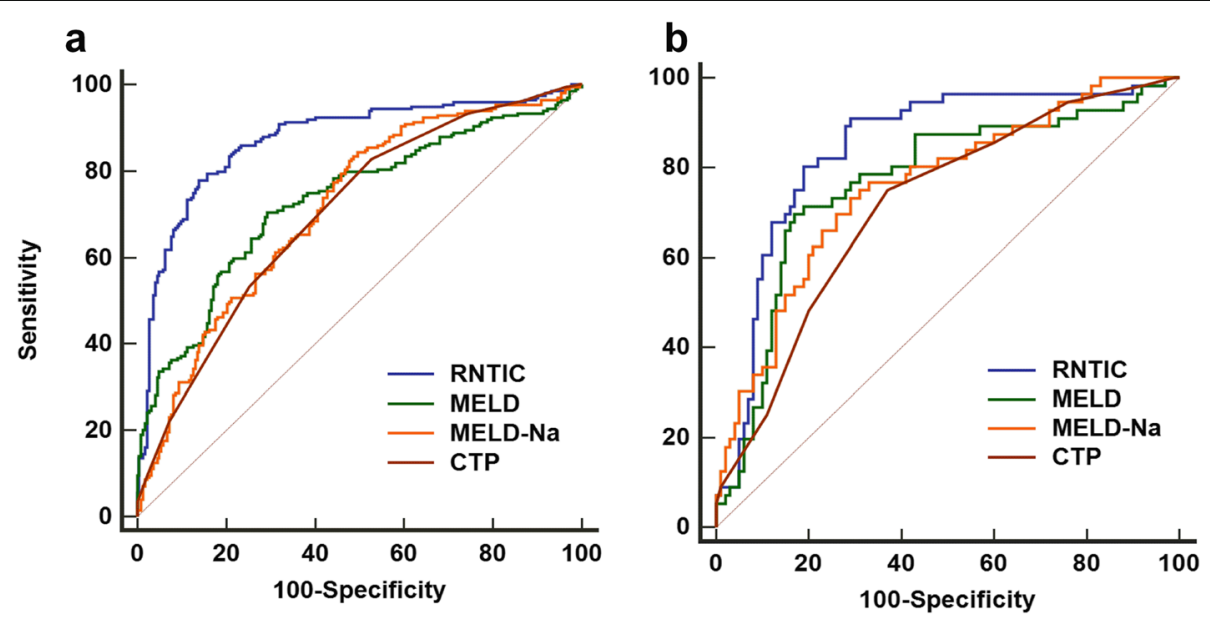

Fig. 3 (a) Receiver operating characteristics (ROC) curve analysis for prediction of 90-day mortality by RNTIC, MELD, MELD-Na and CTP in derivation cohort; (b) Receiver operating characteristics (ROC) curve analysis for prediction of 90-day mortality by RNTIC, MELD, MELD-Na and CTP in validation cohort 
Table 4 Comparisons of demographics and baseline clinical characteristics of the patients in the derivation and validation cohort

\begin{tabular}{|c|c|c|c|}
\hline Variables & Validation cohort $(n=156)$ & derivation cohort $(n=421)$ & $P$ value \\
\hline Age (years) & $48.92 \pm 11.94$ & $47.93 \pm 11.40$ & 0.372 \\
\hline Gender (M: F) & $129 / 27$ & $365 / 56$ & - \\
\hline Cirrhosis (\%) & 114(73.1\%) & $299(71.02 \%)$ & 0.627 \\
\hline Bacterial infections & $101(64.74 \%)$ & $307(72.92 \%)$ & 0.055 \\
\hline TBIL $(\mu \mathrm{mol} / \mathrm{L})$ & $239.43 \pm 136.17$ & $323.39 \pm 165.69$ & $<0.001$ \\
\hline INR & $1.92(1.58,2.43)$ & $2.25(1.86,2.76)$ & $<0.001$ \\
\hline $\mathrm{Cr}(\mu \mathrm{mol} / \mathrm{L})$ & $62.40(52.15,80.35)$ & $69.30(57.28,87.90)$ & 0.004 \\
\hline RDW (\%) & $16.10(14.50,18.10)$ & $15.90(14.38,18.42)$ & 0.842 \\
\hline MPV (FL) & $11.29 \pm 1.67$ & $11.74 \pm 1.53$ & 0.003 \\
\hline NLR & $4.33(2.68,7.87)$ & $4.84(3.19,8.07)$ & $<0.001$ \\
\hline MLR & $0.61(0.41,0.90)$ & $0.64(0.42,0.94)$ & 0.473 \\
\hline GPR & $0.91(0.53,1.50)$ & $0.97(0.59,1.69)$ & 0.162 \\
\hline RPR & $0.17(0.11,0.28)$ & $0.19(0.12,0.30)$ & 0.093 \\
\hline MPR & $0.70(0.59,0.83)$ & $0.14(0.09,0.20)$ & $<0.001$ \\
\hline $\mathrm{PNI}$ & $35.03(30.65,41.10)$ & $34.10(30.20,38.31)$ & 0.033 \\
\hline PLR & $95.92(63.99,143.22)$ & $91.03(62.69,127.69)$ & 0.206 \\
\hline RLR & $17.77(11.73,26.73)$ & $16.70(3.71,85.81)$ & 0.939 \\
\hline MELD SCORE & $21.41 \pm 7.84$ & $25.05 \pm 6.75$ & $<0.001$ \\
\hline MELD-Na & $21.24 \pm 15.66$ & $24.32 \pm 12.69$ & 0.001 \\
\hline CTP & $10.48 \pm 1.89$ & $11.09 \pm 2.00$ & 0.029 \\
\hline
\end{tabular}

TBIL total bilirubin, ALB albumin, $\gamma$-GGT gamma-glutamyl transpeptidase, $C r$ creatinine, Cyst-c Cystatin c, Serum Na + serum sodium, PT prothrombin time, INR international normalized ratio, PTA prothrombin activity, WBC white blood cell count, $R D W$ red blood cell distribution width, NLR neutrophil/lymphocyte ratio, MLR monocyte/lymphocyte ratio, $P L R$ platelet/lymphocyte ratio, RPR RDW/platelet ratio, GPR gamma-glutamyl transpeptidase/platelet ratio, MPV mean platelet volume, $R L R$ RDW/lymphocyte ratio, PNI prognostic nutritional index, MPR MPV/platelet ratio, MELD model for end-stage liver disease, MELD-Na MELD-sodium score, CTP child-Turcotte Pugh score

maturation of red blood cells and cause immature red blood cells to enter the bloodstream simultaneously, leading to an increase in RDW [8]. (II) Inflammatory cytokines such as tumor necrosis factor TNF- $\alpha$, IL- $1 \beta$ and IL-6 may inhibit iron metabolism and erythropoietin production, leading to synthetic disorders or abnormal erythropoietin activity [31]. (III) Excessive hepatocyte necrosis resulting in decreased liver reservation of vitamin B12, folic acid and iron [32], elevated the RDW. (IV) Pathological immune response to $\mathrm{HBV}$ can release inflammatory mediators and endotoxin etc., which affect the growth and development of red blood cells, making RDW rise [8]. (V) Low serum antioxidant concentrations characterized by a compromise between oxidant and antioxidant defenses are associated with increased levels of RDW, which is common in liver disease [33].

Apart from the inflammatory markers NLR and RDW, we also found that Cr, TBIL, and INR, reflecting the function of liver, kidney, and coagulation in routine hematological tests, were independent risk factors for prognosis of HBV-ACLF in this study. Therefore, the new prediction Cox regression model was constructed based on the above five indicators, which showed a great predictive performance both in the derivation and validation cohort with high sensitivity and specificity.

Some limitations of our study must be considered. First, this was a retrospective study, so we did not observe the changes of RDW and NLR values dynamically. In the future, more prospective studies are needed to reveal the association between RDW, NLR longitudinal changes and outcomes in HBV-ACLF patients. Second, we did not test other pro-inflammatory cytokines, such as TNF- $\alpha$, IL- $1 \beta$, IL-6, and IL-8, which may contribute to revealing the mechanisms. Third, the study only analyzed the patients hospitalized in ward and had not analyzed the patients on dialysis under mechanical ventilation. Forth, due to the retrospective study design, some data lacking led that kinds of scoring systems for ACLF such as APASL ACLF Research Consortium (AARC), the Chinese Group on the Study of Severe Hepatitis $\mathrm{B}(\mathrm{COSSH})$ and CIIF-SOFA cannot be analyzed.

\section{Conclusions}

In summary, compared with the MELD score, MELD-Na and CTP, our newly established model has a better predictive ability to assess the 90-day mortality in HBV-ACLF patients in the early stage. 


\section{Supplementary information}

Supplementary information accompanies this paper at https://doi.org/10. 1186/s12876-020-01437-2.

Additional file 1: Additional Table 1. The Comparison of 28-day with 90-day predictive value of RNTIC, MELD, MELD-Na and CTP in derivation cohort. Z Statistic: compared with AUC of RNTIC, $P$ value: compared with $\mathrm{AUC}$ of RNTIC, RNTIC $=0.053 \times \mathrm{RDW}+0.027 \times \mathrm{NLR}+0.003 \times \mathrm{TBIL}+0.317 \times$ $\mathrm{INR}+0.003 \times \mathrm{Cr}, \mathrm{MELD}=$ model for end-stage liver disease, $C \mathrm{TP}=$ childTurcotte Pugh score, MELD-Na = MELD-sodium score, NLR = neutrophil/ lymphocyte ratio, $\mathrm{RDW}=$ red blood cell distribution width, $\mathrm{TBIL}=$ total bilirubin, $\mathrm{Cr}=$ creatinine, INR = international normalized ratio, HBV-ACLF = hepatitis $B$ virus related acute-on-chronic liver failure, NPV = negative predictive value, PPV = positive predict value

Additional file 2: Additional Figure 1. Receiver operating characteristics (ROC) curve analysis for prediction of 28-day mortality by RNTIC, MELD, MELD-Na and CTP in derivation cohort.

\section{Abbreviations}

NLR: neutrophil/lymphocyte ratio; RDW: red blood cell distribution width; HBV-ACLF: hepatitis B virus-related acute-on-chronic liver failure; AUC: the area under the receiver operating characteristics curve; CLIF-SOFA: chronic liver failure sequential organ failure assessment; CTP: child-Turcotte Pugh score; MELD: model for end-stage liver disease score; MELD-Na: MELDsodium score; HBV: hepatitis B virus; MPV: mean platelet volume; MLR: monocyte/lymphocyte ratio; PLR: platelet/lymphocyte ratio; RPR: RDW/ platelet ratio; GPR: gamma-glutamyl transpeptidase/platelet ratio; RLR: RDW/ lymphocyte ratio; PNI: prognostic nutritional index; MPR: MPV/platelet ratio; IL-6: Interleukin-6; TBIL: total bilirubin; INR: international normalized ratio of prothrombin time; PTA: prothrombin activity; ROC: receiver operating characteristic analysis; ALT: alanine aminotransferase; AST: aspartate aminotransferase; ALB: albumin; $\gamma$-GGT: gamma-glutamyl transpeptidase; Cr: creatinine; Cyst-c: cystatin c; Serum $\mathrm{k}^{+}$: serum potassium; Serum $\mathrm{Na}^{+}$: serum sodium; PCT: procalcitonin; HE: Hepatic encephalopathy; NPV: negative predictive value; PPV: positive predict value; AARC: APASL ACLF Research Consortium; COSSH: the Chinese Group on the Study of Severe Hepatitis B

\section{Acknowledgements}

Not applicable.

\section{Authors' contributions}

LQ: collected and analyzed data, designed and wrote the first draft, and revised each version of the manuscript. JQ: collected data and tracked the survival of the patients. CFS, YJS and WC: revised the manuscript. GW: Conception and design, construction of the framework, revision of the manuscript. BDQ and XC: checking, and made the tables and figures, revised the manuscript. YFC and FL: Data collection. All authors have read and approved the manuscript.

\section{Funding}

This work was supported by the Science and Technology Project of the Health department of Sichuan (120326), Collaborative Fund of Luzhou Government and Southwest Medical University (2018LZXNYD-ZK29), Collaborative Fund of Luzhou Government and Sichuan Medical University (2015SX-W35), the Youth Fund of Southwest Medical University (2017-ZRQN103), the Science and Technology Project of the Health Planning Committee of Sichuan (18PJ340), the Ph.D. Research Fund of the Affiliated Hospital of Southwest Medical University (16237). The recipients of the fund, Changfeng Sun revised the manuscript and Gang WU designed the study, constructed the framework and revised the manuscript.

\section{Availability of data and materials}

The datasets analyzed during the current study are not publicly available because they contain sensitive patient information, but may available from the corresponding author on reasonable request.

\section{Ethics approval and consent to participate}

The study was approved by the Institutional Ethics Committee of Affiliated Hospital of Southwest Medical University and performed in adherence with the principles of the Declaration of Helsinki. Written informed consent was obtained from the patients and at the case of the patient was unable to write or the patient was unconscious, it was obtained from their family members.

\section{Consent for publication}

Not applicable.

\section{Competing interests}

The authors declared no conflicts of interest.

\section{Author details}

${ }^{1}$ Department of Infectious disease, The Affiliated Hospital of Southwest Medical University, Luzhou City 646000, Sichuan Province, China.

${ }^{2}$ Department of Infectious disease, Public Health Clinical Center of Chengdu, Chengdu City 610000, Sichuan Province, China. ${ }^{3}$ Department of Infectious disease, The Second People's Hospital of Yibin, Yibin City 644000, Sichuan Province, China. ${ }^{4}$ Department of Infectious disease, The First People's Hospital of Neijiang, Neijiang City 641000, Sichuan Province, China.

Received: 8 August 2019 Accepted: 28 August 2020

Published online: 16 September 2020

\section{References}

1. Sarin SK, Kedarisetty CK, Amarapukar D, et al. Acute-on-chronic liver failure: consensus recommendations of the Asian Pacific Association for the Study of the liver (APASL) 2014. Hepatol Int. 2014;8(4):453-71.

2. Volarevic $V$, Markovic BS, Gazdic M, et al. Ethical and safety issues of stem cell-based therapy. Int J Med Sci. 2018;15(1):36-45.

3. Besch C, Michard B, Addeo $P$, et al. Impact of early remote organ dysfunction on long-term survival after liver transplantation. Clin Res Hepatol Gastroenterol. 2019;43(6):730-7.

4. Antunes AG, Teixeira $C$, Vaz AM, et al. Comparison of the prognostic value of chronic liver failure consortium scores and traditional models for predicting mortality in patients with cirrhosis. Gastroenterol Hepatol. 2017; 40(4):276-85.

5. Premkumar M, Saena $P$, Rangeowda D, et al. Coagulation failure is associated with bleeding events and clinical outcome during systemic inflammatory response and sepsis in acute-on-chronic liver failure: an observational cohort study. Liver Int. 2019;39(4):694-704.

6. Trebicka J, Amoros A, Pitarch C, et al. Addressing profiles of systemic inflammation across the different clinical phenotypes of acutely decompensated cirrhosis. Front Immunol. 2019;10:476.

7. Clària J, Stauber RE, Coenraad MJ, et al. Systemic inflammation in decompensated cirrhosis. Characterization and role in acute-on-chronic liver failure. Hepatology. 2016;64(4):1249-64.

8. Cetinkaya E, Senol K, Saylam B, et al. Red cell distribution width to platelet ratio: new and promising prognostic marker in acute pancreatitis. World J Gastroenterol. 2014;20(39):14450-4.

9. Bendall LJ, Bradstock KF. G-CSF: from granulopoietic stimulant to bone marrow stem cell mobilizing agent. Cytokine Growth Factor Rev. 2014;25(4): 355-67.

10. Kaser A, Brandacher G, Steurer W, et al. Interleukin-6 stimulates thrombopoiesis through thrombopoietin: role in inflammatory thrombocytosis. Blood. 2001;98(9):2720-5.

11. Ceylan B, Fincanci M, Yardimci C, et al. Can mean platelet volume determine the severity of liver fibrosis or inflammation in patients with chronic hepatitis B? Eur J Gastroenterol Hepatol. 2013;25(5):606-12.

12. Liu $H$, Zhang $H$, Wan $G$, Sang $Y$, et al. Neutrophil-lymphocyte ratio: a novel predictor for short-term prognosis in acute-on-chronic hepatitis B liver failure. J Viral Hepat. 2014;21(7):499-507.

13. Karagöz E, Tanoğlu A, Ülçay A, et al. Mean platelet volume and red cell distribution width to platelet ratio for predicting the severity of hepatic fibrosis in patients with chronic hepatitis C. Eur J Gastroenterol Hepatol. 2016;28(7):744-8.

14. Wu J, Zhang $X$, Liu $H$, et al. RDW, NLR and RLR in predicting liver failure and prognosis in patients with hepatitis $E$ virus infection. Clin Biochem. 2019;63:24-31.

15. Cai J, Wang K, Han T, Jiang H. Evaluation of prognostic values of inflammation-based makers in patients with HBV-related acute-on-chronic liver failure. Medicine (Baltimore). 2018;97(46):e13324. 
16. Liver Failure and Artificial Liver Group, Chinese Society of Infectious Diseases, Chinese Medical Association; Severe Liver Disease and Artificial Liver Group, Chinese Society of Hepatology. Chinese Medical Association Guideline for diagnosis and treatment of liver failure. Zhonghua Gan Zang Bing Za Zhi. 2019;27:18-26.

17. Ferenci P, Lockwood A, Mullen K, et al. Hepatic encephalopathy—definition, nomenclature, diagnosis, and quantification: final report of the working party at the 11th world congresses of gastroenterology, Vienna, 1998. Hepatology. 2002;35(3):716-21.

18. Kamath PS, Kim WR. The model for end-stage liver disease (MELD). Hepatology. 2010;45(3):797-805.

19. Biggins SW, Kim WR, Terrault NA, et al. Evidence-Based Incorporation of Serum Sodium Concentration Into MELD. Digest of the World Core Medical Journals(Gastroenterology). 2006;130(6):1652-60.

20. Zhou C, Hou C, Cheng D, et al. Predictive accuracy comparison of MELD and child-Turcotte-Pugh scores for survival in patients underwent TIPS placement: a systematic meta-analytic review. Int J Clin Exp Med. 2015;8(8): 13464-72.

21. Bernardi M, Moreau R, Angeli P, et al. Mechanisms of decompensation and organ failure in cirrhosis: from peripheral arterial vasodilation to systemic inflammation hypothesis. J Hepatol. 2015;63(5):1272-84.

22. Moreau R. The pathogenesis of ACLF: the inflammatory response and immune function. Semin Liver Dis. 2016;36(2):133-40.

23. Rocha NP, Fortes RC. Total lymphocyte count and serum albumin as predictors of nutritional risk in surgical patients. Arq Bras Cir Dig. 2015; 28(3):193-6.

24. Yoshitomi R, Nakayama M, Sakoh T, et al. High neutrophi//lymphocyte ratio is associated with poor renal outcomes in Japanese patients with chronic kidney disease. Ren Fail. 2019;41(1):238-43.

25. Sargin G, Senturk T, Yavasoglu I, et al. Relationship between neutrophillymphocyte, platelet-lymphocyte ratio and disease activity in rheumatoid arthritis treated with rituximab. Int J Rheum Dis. 2018;21(12):2122-7.

26. Peng $Y, L i Y$, Wei $Q$, et al. The role of neutrophil to lymphocyte ratio for the assessment of liver fibrosis and cirrhosis: a systematic review. Expert Rev Gastroenterol Hepatol. 2018;12(5):503-13.

27. Wong L, Bozhilov K, Hernandez B, et al. Underlying liver disease and advanced stage liver cancer are associated with elevated neutrophillymphocyte ratio. Clin Mol Hepatol. 2019;25(3):305-16.

28. Albillos A, Lario M, Alvarez-Mon M. Cirrhosis-associated immune dysfunction: distinctive features and clinical relevance. J Hepatol. 2014;61(6): 1385-96.

29. Kwon JH, Jang JW, Kim YW, et al. The usefulness of C-reactive protein and neutrophil-to-lymphocyte ratio for predicting the outcome in hospitalized patients with liver cirrhosis. BMC Gastroenterol. 2015;15:146.

30. Lippi GM, Plebani M. Red blood cell distribution width (RDW) and human pathology. One size fits all. Clin Chem Lab Med. 2014;52(9):1247-9.

31. Semba RD, Patel KV, Ferrucci $L$, et al. Serum antioxidants and inflammation predict red cell distribution width in older women: the Women's health and aging study I. Clin Nutr. 2010;29(5):600-4.

32. Tefferi A. Anemia in adults: a contemporary approach to diagnosis. Mayo Clin Proc. 2003;78(10):1274-80.

33. Friedman JS, Lopez MF, Fleming MD, et al. SOD2-deficiency anemia: protein oxidation and altered protein expression reveal targets of damage, stress response, and antioxidant responsiveness. Blood. 2004;104(8):2565-73.

\section{Publisher's Note}

Springer Nature remains neutral with regard to jurisdictional claims in published maps and institutional affiliations.

Ready to submit your research? Choose BMC and benefit from:
- fast, convenient online submission
- thorough peer review by experienced researchers in your field
- rapid publication on acceptance
- support for research data, including large and complex data types
- gold Open Access which fosters wider collaboration and increased citations
- maximum visibility for your research: over 100M website views per year
At BMC, research is always in progress.
Learn more biomedcentral.com/submissions

